This is an electronic reprint of the original article. This reprint may differ from the original in pagination and typographic detail.

Author(s): Koivisto, Hannu; Suominen, Pekka; Tarvainen, Olli; Spädtke, P.

Title: $\quad$ The electron cyclotron resonance ion source with arc-shaped coils concept

Year: $\quad 2012$

Version:

Please cite the original version:

Koivisto, H., Suominen, P., Tarvainen, O., \& Spädtke, P. (2012). The electron cyclotron resonance ion source with arc-shaped coils concept. Review of Scientific Instruments, 83(2), 02A312. https://doi.org/10.1063/1.3662474

All material supplied via JYX is protected by copyright and other intellectual property rights, and duplication or sale of all or part of any of the repository collections is not permitted, except that material may be duplicated by you for your research use or educational purposes in electronic or print form. You must obtain permission for any other use. Electronic or print copies may not be offered, whether for sale or otherwise to anyone who is not an authorised user. 


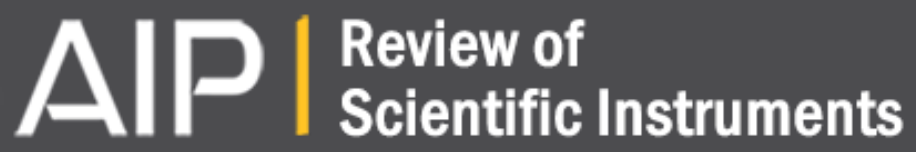

The electron cyclotron resonance ion source with arc-shaped coils concept (invited)a)

H. Koivisto, P. Suominen, O. Tarvainen, and P. Spädtke

Citation: Review of Scientific Instruments 83, 02 A312 (2012); doi: 10.1063/1.3662474

View online: http://dx.doi.org/10.1063/1.3662474

View Table of Contents: http://scitation.aip.org/content/aip/journal/rsi/83/2?ver=pdfcov

Published by the AIP Publishing

\section{Articles you may be interested in}

Charge breeding results and future prospects with electron cyclotron resonance ion source and electron beam ion source (invited)a)

Rev. Sci. Instrum. 83, 02A913 (2012); 10.1063/1.3673629

Concept for a fourth generation electron cyclotron resonance ion sourcea)

Rev. Sci. Instrum. 83, 02 A301 (2012); 10.1063/1.3655527

A status report of the multipurpose superconducting electron cyclotron resonance ion source (invited)a)

Rev. Sci. Instrum. 79, 02 A326 (2008); 10.1063/1.2812336

Electron cyclotron resonance ion sources with arc-shaped coilsa)

Rev. Sci. Instrum. 79, 02A305 (2008); 10.1063/1.2805209

Results with the superconducting electron cyclotron resonance ion source VENUS (invited)

Rev. Sci. Instrum. 75, 1389 (2004); 10.1063/1.1675927






\title{
The electron cyclotron resonance ion source with arc-shaped coils concept (invited) $)^{\text {a) }}$
}

\author{
H. Koivisto, ${ }^{1, b)}$ P. Suominen, ${ }^{2}$ O. Tarvainen, ${ }^{1}$ and P. Spädtke ${ }^{3}$ \\ ${ }^{1}$ Department of Physics, University of Jyväskylä, Jyväskylä, Finland \\ ${ }^{2}$ CERN, CH-1211 Genève 23, Switzerland \\ ${ }^{3}$ GSI, Darmstadt, Planckstr. 1, 64221 Darmstadt, Germany
}

(Presented 13 September 2011; received 7 September 2011; accepted 4 October 2011; published online 6 February 2012)

The main limitation to further improve the performance of ECR ion sources is set by the magnet technology related to the multipole magnet field used for the closed minimum-B structure. The JYFL ion source group has sought different approaches to improve the strength of the minimum-B structure required for the production of highly charged ion beams. It was found out that such a configuration can be realized with arc shaped coils. The first prototype, electron cyclotron resonance ion source with arc-shaped coils (ARC-ECRIS), was constructed and tested at JYFL in 2006. It was confirmed that such an ion source can be used for the production of highly charged ion beams. Regardless of several cost-driven compromises such as extraction mirror ratio of 1.05-1.2, microwave frequency of $6.4 \mathrm{GHz}$, and beam line with limited capacity, $\mathrm{Ar}^{4+}$ beam intensity of up to $2 \mu \mathrm{A}$ was measured. Subsequent design study has shown that the ARC-ECRIS operating at the microwave frequency above $40 \mathrm{GHz}$ could be constructed. This specific design would be based on NbTi-wires and it fulfills the experimental magnetic field scaling laws. In this article, the ARC-ECRIS concept and its potential applications will be described. ( 2012 American Institute of Physics. [doi:10.1063/1.3662474]

\section{INTRODUCTION}

Electron cyclotron resonance ion sources (ECRIS) (Ref. 1) are widely used for the production of intense, highly charged ion beams, e.g., for research in nuclear and material physics, material modifications, and medical purposes. A comprehensive list of various applications has been presented for example in Ref. 2.

The magnetic field of an ECRIS is so-called minimumB structure in which electrons are heated on a closed surface where the magnetic field strength fulfills the electron cyclotron resonance condition $\mathrm{B}_{\mathrm{ECR}}=m_{e} \omega_{\mathrm{RF}} / e$. The structure can be produced with a combination of solenoid and multipole magnetic fields (normally hexapole/sextupole).

Intensive research and development work to improve the understanding of ECRIS parameters affecting the capability of ion source to produce highly charged ion beams with adequate ion beam quality has been carried out by a number of research groups. It has been found out, for example, that the magnetic field configuration and the microwave frequency have a strong impact on the performance of an ECRIS. The microwave frequency affects the heating efficiency and density of electrons and magnetic field affects the plasma confinement.

It is a well-known fact that the beam intensity of highly charged ion beams has a tendency to increase with the microwave frequency used for the electron heating. It is generally accepted that the tendency is proportional to the square of the heating frequency $\omega$, i.e., $I \propto \omega^{2}$. The dependence

\footnotetext{
a) Invited paper, published as part of the Proceedings of the 14th International Conference on Ion Sources, Giardini Naxos, Italy, September 2011.

b)Electronic mail: hannu.koivisto@phys.jyu.fi.
}

can be demonstrated (roughly) by comparing various ion sources with different heating frequencies (see, for example, Refs. 2 and 3). There are also other parameters affecting the performance of ion source, e.g., plasma volume, vacuum conditions, plasma chamber material, and microwave power density. These parameters can naturally cause a discrepancy to the frequency scaling.

As a result of frequency scaling, as high microwave frequency as possible is desired. However, the magnetic field has to be increased with the microwave frequency used for electron heating. Furthermore, certain magnetic field configuration is needed in order to maximize the ion beam intensity of highly charged ions. The optimal magnetic field structure has mainly been studied with SC-ECRIS at NSCL/MSU and SERSE at LNS-INFN. ${ }^{3,4}$ As a result of this work following rules for the optimum magnetic fields have been given,

$$
\frac{\mathrm{B}_{\mathrm{inj}}}{\mathrm{B}_{\mathrm{ECR}}}=4, \frac{\mathrm{B}_{\mathrm{rad}}}{\mathrm{B}_{\mathrm{ECR}}}=2 \quad \mathrm{~B}_{\min } \approx 0.4 \mathrm{~B}_{\mathrm{rad}} \text { and } \mathrm{B}_{\mathrm{ext}} \approx 0.9 \mathrm{~B}_{\mathrm{rad}},
$$

where $B_{E C R}$ is the magnetic field on the resonance and $B_{\min }$ the minimum magnetic field at the center of the plasma chamber. The fields $B_{\text {inj }}, B_{\text {ext }}$, and $B_{\text {rad }}$ refer to magnetic fields at the injection side, extraction side, and multipole field, respectively. The aforementioned work shows also the strong dependence of beam intensity as a function of mirror ratios. For example, the intensity of $\mathrm{Xe}^{20+}$ ion beam produced by SERSE using $18 \mathrm{GHz}$ or $28 \mathrm{GHz}$ heating mode increased about sixfold when the radial mirror ratio increased from 1.2 to 2 . The scaling rules obtained for the magnetic fields have been used as a basis when more powerful ECR ion sources have been designed. 

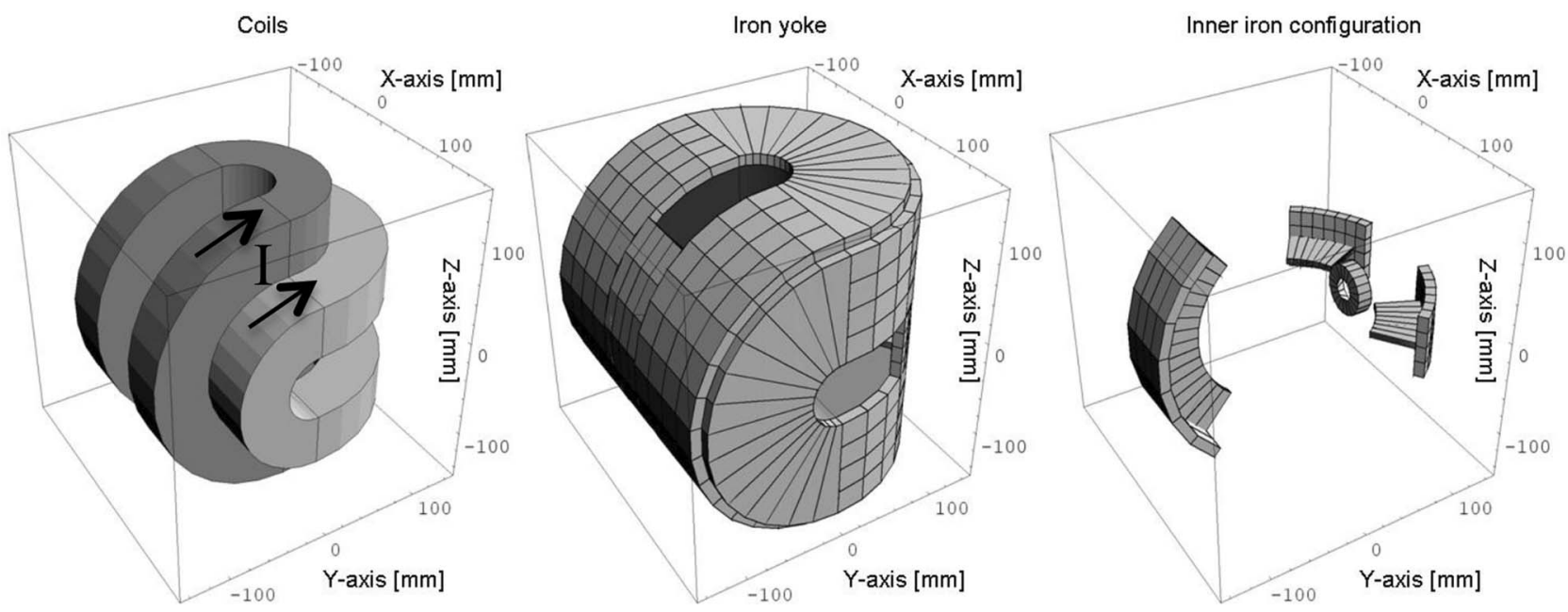

FIG. 1. The magnetic field configuration of the ARC-ECRIS is produced by using arc-shaped coils. The magnetic field can be optimized with the aid of iron yoke and inner iron parts. ${ }^{9}$

The most powerful ECR ion sources, such as VENUS, ${ }^{5}$ use the heating frequency of about $28 \mathrm{GHz}$. These so-called 3 rd generation ECR ion sources are fully superconducting and are realized using NbTi wires. The most critical part of the device is rather complicated sextupole structure inside a strong solenoid magnetic field. A special attention has to be paid on the clamping of magnets in order to prevent any movement caused by the enormous magnetic forces between the excited wires. An alternative approach to minimizing magnetic forces has been developed and successfully used at IMP/HIRFL with SECRAL ECR ion source. ${ }^{6}$ In this scheme, the sextupole structure is located outside the solenoids and their field, which minimizes the magnetic field and forces affecting the superconducting wires. However, this inverted geometry requires very large and strong multipole in order to reach the required field strength on the inner surface of plasma chamber.

A comprehensive design study about the possibilities of constructing a 4th generation ECR ion source has been performed by the LBNL ion source group. ${ }^{7}$ This ECRIS would operate at the microwave frequency of about $56 \mathrm{GHz}$ requiring the resonance field of $2 \mathrm{~T}$. According to the scaling rules the multipole field of $4 \mathrm{~T}$ is required. The work presented shows that the 4th generation ECR ion source meeting the magnetic field scaling rules can be realized using $\mathrm{NbSn}_{3}$ conductor. The work also presents the means to prevent conductor motions. ${ }^{8}$

The JYFL ion source group has proposed and tested an alternative scheme to produce the minimum-B structure without the multipole. The approach significantly simplifies the structure and consequently could minimize the problems relating to the complicated magnetic field configuration. This new type of ECR ion source is called electron cyclotron resonance ion source with arc-shaped coils (ARC-ECRIS) (Ref. 9) and will be described in this article.

\section{THE PROTOTYPE OF ARC-ECRIS}

This alternative minimum-B type magnetic field configuration has been tested for fusion experiments using "yin-yang" type coils. The objective of the work was to heat light ions, such as hydrogen and helium, towards the temperature needed for fusion studies (see, for example, Refs. 9 and 10 for further information and references therein). It was noticed that the scheme was not adequate for the purpose because ions remained cold. However, this property is desirable when ion beams of highly charged ions are produced. Because of this, the proof-of-principle prototype was constructed and tested at JYFL in order to study this interesting option to produce the B-minimum-type magnetic field configuration.

The prototype was designed for the microwave frequency of $6.4 \mathrm{GHz}$ in order to reduce costs. Consequently, the power supplies, microwave system, and other auxiliary devices of the JYFL 6.4 GHz ECRIS were used. In addition, the simple beam line equipped with the salvaged cast-off NMR dipole magnet was constructed for the mass separation. Two ARCshaped coils, as shown in Fig. 1, were designed and wounded for the prototype. As the figure shows the ARC-coil can be considered as a normal racetrack coil, which has been bent. The magnetic field configuration inside the plasma chamber was adjusted using iron yoke and several iron parts also shown in Fig. 1. Regardless of this the magnetic field scaling rules shown by Eq. (1) were not fulfilled. This is demonstrated in Fig. 2, which shows the magnetic field profile of the prototype along different axis. The maximum mirror ratio in location of the extraction aperture was around 1.25. However, in this case the distance between the resonance points was only $8 \mathrm{~mm}$. During the tests the aforementioned mirror ratio varied between 1.05 and 1.2. In the case of conventional ECR ion source such a low mirror ratio would result in very poor performance. An excellent demonstration concerning the effect of mirror ratio to the beam currents can be found in Ref. 4.

Figure 3 shows the construction of the prototype. The other ARC-coil has been removed in order to visualize the plasma chamber. The outer diameter of the spherical plasma chamber is $120 \mathrm{~mm}$. It has four identical $50 \mathrm{~mm}$ feedthroughs; three horizontal and one vertical. The feedthrough on the lefthand side is used for microwave and gas injection, the center 


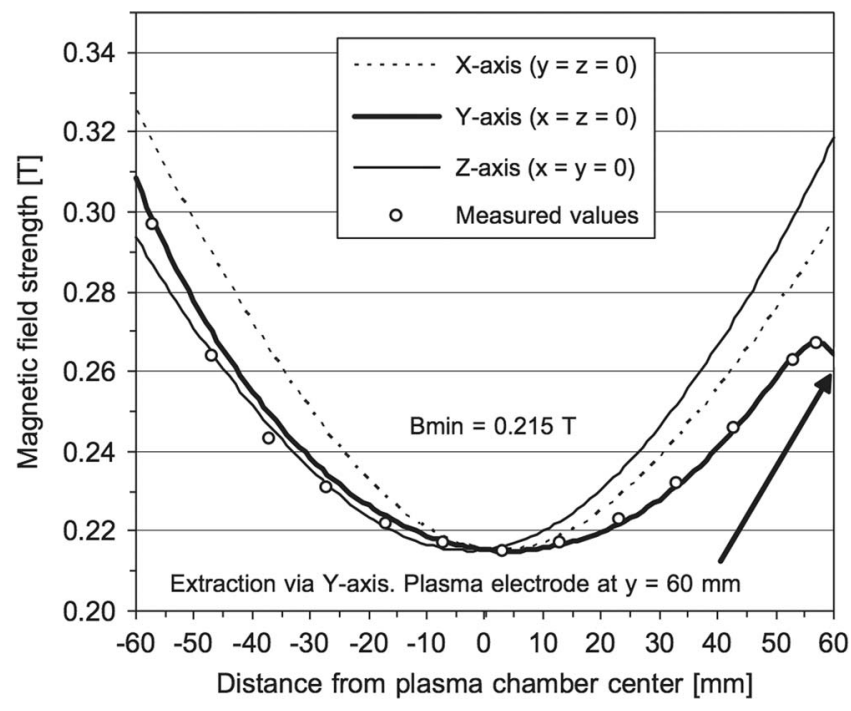

FIG. 2. The magnetic field profile of the prototype on different axis (Ref. 9).

one for extraction and one on the right-hand side for vacuum pumping and as a viewport.

The beam was extracted through the extraction aperture of $6 \mathrm{~mm}$ (in diameter) using the acceleration voltage of $9 \mathrm{kV}$. Due to the low resolving power of dipole $\left(45^{\circ}\right.$ angle, $240 \mathrm{~mm}$ radius) the ion beam was measured with the Faraday cup of $5 \mathrm{~mm}$ in diameter. The experiments were performed with the argon plasma and no gas mixing was used. Bias disk was shortly tested but its effect to the beam intensity was negligible. Instead, very high current from the bias disk $(30 \mathrm{~mm}$ in diameter), exceeding the power supply limit of $10 \mathrm{~mA}$ at $-10 \mathrm{~V}$, was measured. Regardless of poor magnetic confinement the beam intensity of $1.1 \mu \mathrm{A}$ for $\mathrm{Ar}^{4+}$ ion beam was reached at the microwave power of only $30 \mathrm{~W}$. In this case mirror ratio was about 1.2 and the length between the resonances was about $45 \mathrm{~mm}$. The intensity of around $2 \mu \mathrm{A}$ was reached at the microwave power of around $200 \mathrm{~W}$. It was noticed that the plasma usually became very unstable when increasing the power. The full description of the setup and results has been given in Ref. 9.

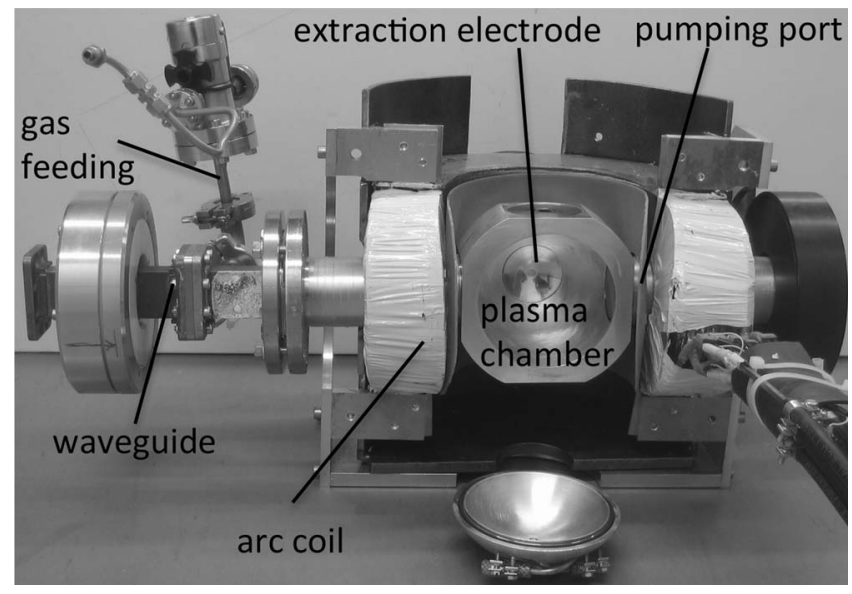

FIG. 3. The first ARC-ECRIS tested at JYFL (Ref. 9).

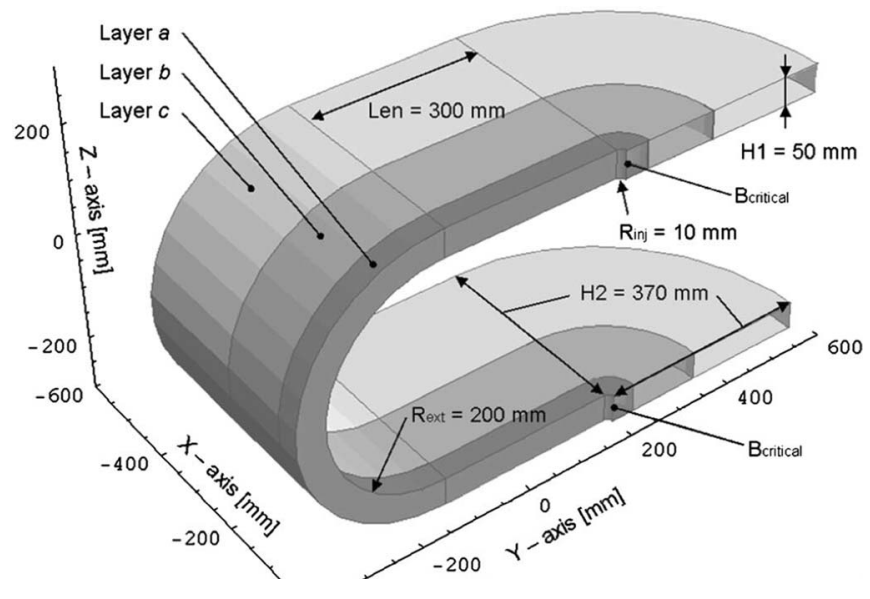

FIG. 4. New ARC-coil and design parameters needed for simulations. (Reprinted with permission from P. Suominen and F. Wenander, Rev. Sci. Instrum. 79, $02 \mathrm{~A} 305$ (2008). Copyright 2008, American Institute of Physics.)

\section{LATEST SIMULATIONS}

\section{A. Magnetic field simulations}

As a result of encouraging proof-of-principle tests the design study for optimized and more powerful ARC-ECRIS was started. ${ }^{11}$ Figure 4 shows the evolution version of the arc-coil and as an example the set of design parameter values used for simulations. In the case of evolution version only one arc-coil is used (notice that the figure shows a cross sectional view of the coil). The coil is divided into three different layers $(a, b$, and $c$ ) and each of them can be excited using different power supply in order to obtain more flexible magnetic field structure. The objective of the work was to design a room temperature ARC-ECRIS using the microwave frequency of at least $14 \mathrm{GHz}$ and fulfilling the scaling rules shown in Eq. (1). Another objective was to design a superconducting ARC-ECRIS using as high operation frequency as possible.

\section{Room temperature ARC-ECRIS}

The latest design study showed that magnetic field configuration up to $18 \mathrm{GHz}$ operation frequency is feasible using the ARC-concept. A possible solution for this heating frequency is achieved when the following design values are used: $\mathrm{R}_{\mathrm{inj}}=30 \mathrm{~mm}, \mathrm{R}_{\mathrm{ext}}=60 \mathrm{~mm}, \mathrm{H} 1=160 \mathrm{~mm}, \mathrm{H} 2=260 \mathrm{~mm}$, and Len $=120 \mathrm{~mm}$. The current density of $10 \mathrm{~A} / \mathrm{mm}^{2}$ was used in all layers resulting in the power consumption of $167 \mathrm{~kW}$. As a comparison the power consumption of the JYFL $14 \mathrm{GHz}$ ECRIS (Ref. 12) is about $75 \mathrm{~kW}$.

The magnetic field configuration achieved using the above-mentioned design parameter values is shown in Fig. 5. Figure 6 shows that the closed minimum-B configuration is indeed produced. The direction of the extracted ion beam is parallel with the y-axis. As Fig. 5 shows the magnetic field in the extraction is slightly below $1 \mathrm{~T}$. The $\mathrm{B}_{\text {min }}$ value is about $0.5 \mathrm{~T}$ being optimal for $18 \mathrm{GHz}$ microwave frequency. The dimensions of the plasma chamber (outer dimensions) are about $35 \mathrm{~cm}, 30 \mathrm{~cm}$, and $12 \mathrm{~cm}$ in $\mathrm{y}, \mathrm{x}$, and $\mathrm{z}$ directions, respectively. The corresponding mirror ratios are approximately: 


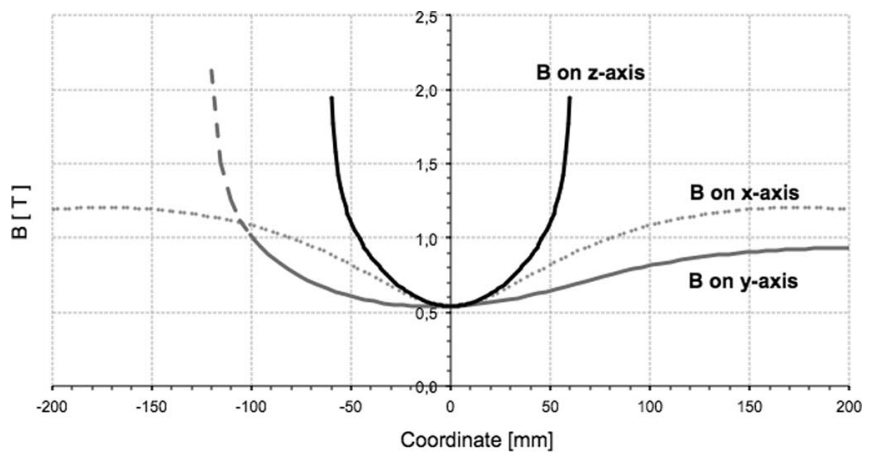

FIG. 5. Magnetic field configuration of the $18 \mathrm{GHz}$ ARC-ECRIS when the design values mentioned above have been used.

1.5 (extraction), 2 (x-axis), and 3 (z-axis). The thickness of the plasma chamber wall has not been taken into account in this study. Additional solenoid coil can be added into the extraction side in order to improve the tuning possibilities of ARC-ECRIS.

\section{Superconducting ARC-ECRIS}

The design parameter values for the superconducting ARC-ECRIS are given in Fig. 4. In this example, the coil layers are divided with a ratio of 1:3:5 resulting in the layer

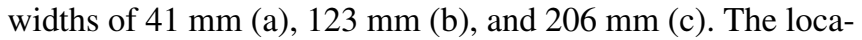
tion of the highest magnetic field load on the superconducting wire is denoted by $B_{\text {critical }}$ (see Fig. 4). Above this value the conductor will lose its superconductivity.

In the simulations the maximum current density values of $\mathrm{NbTi}$ conductor are used. These so called engineering current density values (curves) have been presented, for example, in Refs. 7 and 11. Simulations presented in Ref. 11 show that

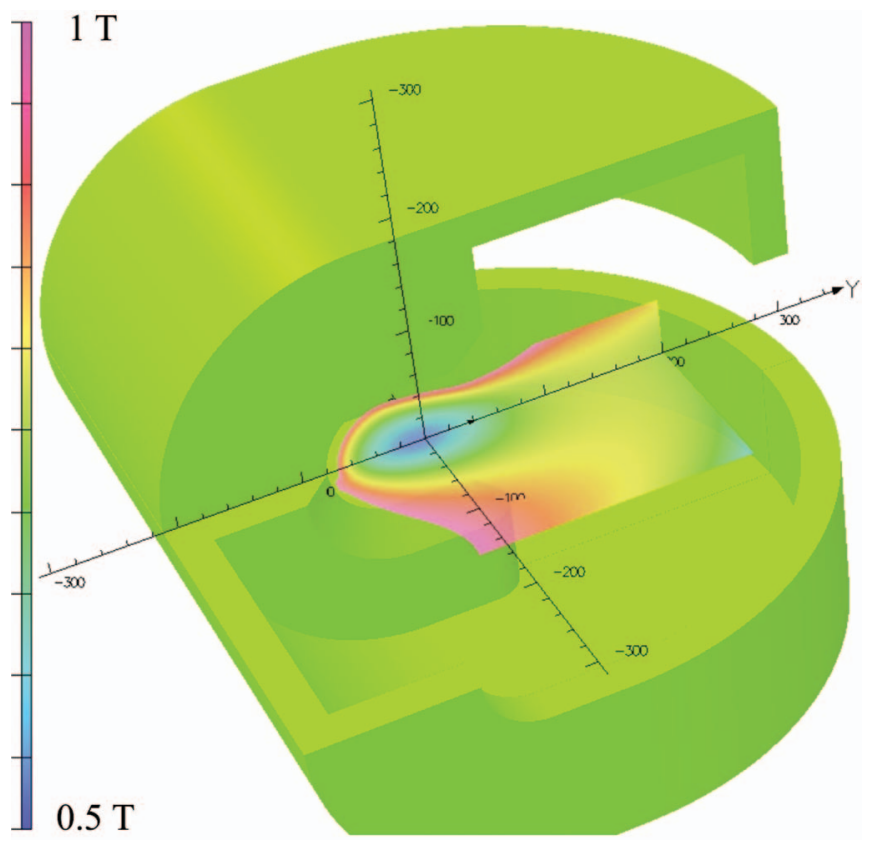

FIG. 6. (Color online) A closed minimum-B-type structure is produced with the ARC-shaped coils (x,y, and $\mathrm{z}$-axis in $\mathrm{mm}$ ). Lowest value of around $0.5 \mathrm{~T}$ is achieved in the center of the plasma chamber.

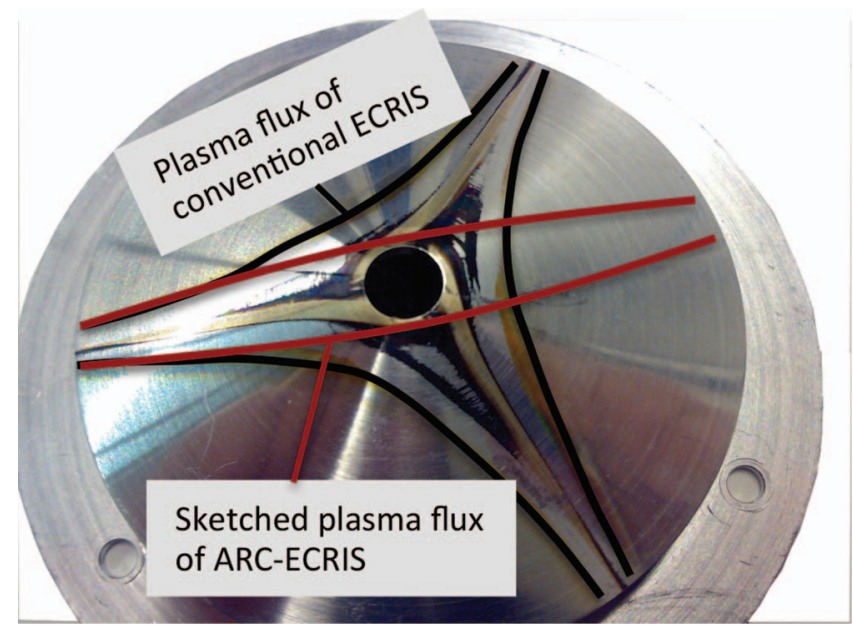

FIG. 7. (Color online) Plasma flux shape of conventional ECRIS and ARCECRIS on the extraction electrode.

the operation frequency of $60 \mathrm{GHz}$, still meeting the magnetic field scaling rules, can be achieved using the arc-coil dimensions shown in Fig. 4. In this example, the current densities of 67,120 , and $250 \mathrm{~A} / \mathrm{mm}^{2}$ are used for the layers a, $\mathrm{b}$, and c, respectively. The critical magnetic field value where superconductivity is lost has not been exceeded at any point of super-conducting wire.

\section{B. Optical simulations}

The plasma shape of the ARC-ECRIS differs strongly from the conventional ECRIS. The conventional ECR ion source produces well-known triangle shape on the plasma electrode as is demonstrated in Fig. 7. The structure originates from the superposition of solenoid and multipole magnetic fields and according to some simulations this structure is also present in the extracted ion beam. ${ }^{13,14}$ In the case of the ARC-ECRIS, a quadrupole like plasma flux shown also in Fig. 7 is generated. No simulations have been performed to compare which scheme produces a higher flux of highly charged ions through the extraction aperture. However, due to the absence of nonlinear effects it can be expected that beam produced by the ARC-ECRIS should have a lower emittance compared to the conventional ECRIS.

Figure 8 shows the simulated beam envelopes after the extraction of the JYFL $14 \mathrm{GHz}$ ECRIS (left side) and

\section{JYFL 14 GHz ECRIS}

\section{ARC-ECRIS}

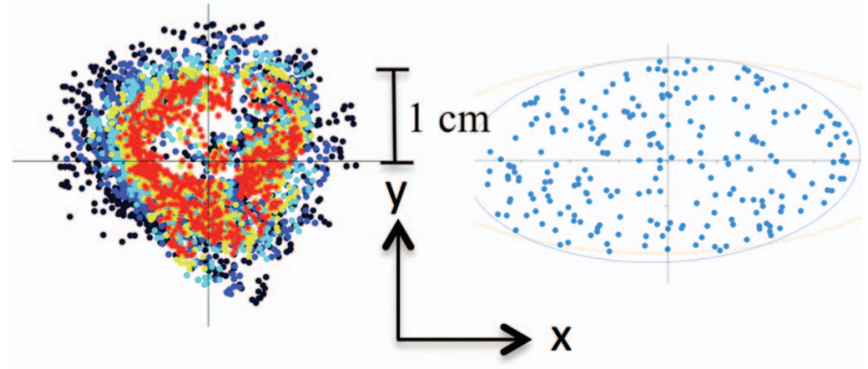

FIG. 8. (Color online) Simulated beam envelopes of the JYFL $14 \mathrm{GHz}$ ECRIS and the ARC-ECRIS. The beam propagates parallel to z-axis. The scale of $1 \mathrm{~cm}$ is shown. 


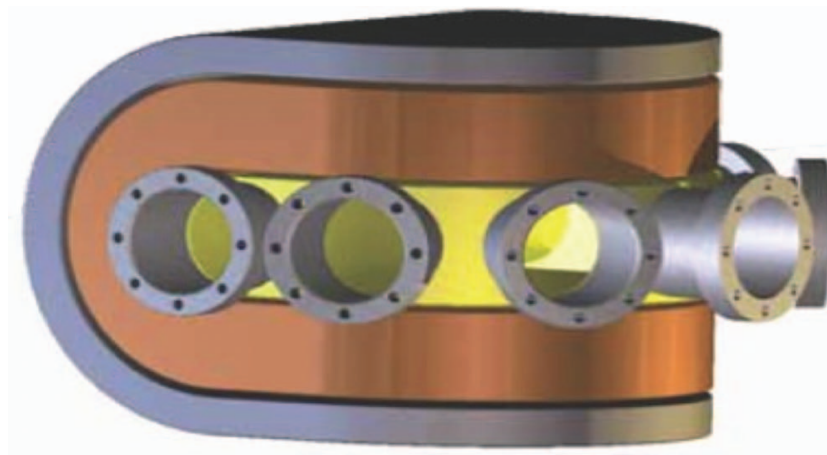

FIG. 9. (Color online) Structure of the ARC-ECRIS leaves space for plasma diagnostics and for techniques required by the metal ion beam production.

the ARC-ECRIS (right side). The simulations have been performed with KOBRA code ${ }^{15}$ and the results show that the structure of the plasma is still present in the extracted ion beams. As a consequence of the simulation results the ARC-ECRIS favors the beam line equipped with quadrupole focusing ${ }^{16}$ resulting in the perfect match between the ion beam properties and ion beam optics.

\section{VERSATILITY OF ARC-ECRIS}

The described room temperature ARC-ECRIS design has some advantages over the conventional ECRIS based on the combination of solenoids and permanent magnet multipole. Two examples will be given: (1) production of stable metal ion beams and (2) production of radioactive ion beams, i.e., charge breeding or beam merging.

In the ARC-ECRIS the minimum-B structure is achieved with a single coil. The simplicity of the structure leaves ample space for inserting ovens and/or sputter probes directly into the plasma chamber. This is demonstrated in Fig. 9. The location of these insertions can be optimized for each technique, e.g., the sputter probe can be placed directly into the plasma flux. Furthermore, the lack of permanent magnets alleviates problems with high temperature operation, which improves the production efficiency of metal ions due to reduced adsorption of atoms on the plasma chamber surfaces. Maximizing the efficiency is especially important when ion beams are produced from expensive isotopes such as ${ }^{48} \mathrm{Ca}$.

The lack of permanent magnets and the simplicity of the structure enable production of radioactive ion beams with ARC-ECRIS. The use of high-grade permanent magnets is unfeasible in high radiation environment. Thus, charge breeder design based on radiation hard materials, such as ARC-ECRIS, opens up possibilities to new experiments with radioactive ions. The simplicity of the structure allows incorporating an injection for $1+$ ions or combining the device with a production target for direct production of $\mathrm{n}+$ ions. Applying ARC-ECRIS for charge breeding requires further studies, e.g., on the injection of the $1+$ ion beams.

A specific example of employing the ARC-ECRIS concept in the production chain of radioactive ion beams has been given in the EURISOL design study. ${ }^{17}$ It has been suggested there that ARC-ECRIS type device with low extraction mirror ratio could be utilized for fast and efficient $(\sim 1 \mathrm{~ms} / 20 \%)$ merging of several radioactive $1+$ ion beams, produced in a multi-MW spallation target, into a single $1+$ ion beam of the given isotope. The beam-merging device is without a doubt one of the most challenging components of the proposed EURISOL facility.

\section{FUTURE PROSPECTS}

The ARC-ECRIS offers very interesting option not only for the production of highly charged ion beams but also for some special techniques such as metal ion beam production and beam merging. The funding for the construction of $14 \mathrm{GHz}$ ARC-ECRIS has actively been sought. The real potential of the ARC-ECRIS concept can be determined only through the realization of more optimized and more powerful design.

\section{ACKNOWLEDGMENTS}

This work has been supported by the Academy of Finland under the Finnish Centre of Excellence Programme 20062011 (Nuclear and Accelerator Based Physics Programme at JYFL) and by the EU 7th framework programme "Integrating Activities - Transnational Access," Project No. 262010 (ENSAR).

${ }^{1}$ R. Geller, Electron Cyclotron Resonance Ion Sources and ECR Plasmas (Institute of Physics, Bristol, 1996).

${ }^{2}$ H. Koivisto, AIP Conf. Proc. 1099, 25 (2009).

${ }^{3}$ D. Hitz, A. Girard, G. Melin, S. Gammino, G. Ciavola, and L. Celona, Rev. Sci. Instrum. 73(2), 509 (2002).

${ }^{4}$ S. Gammino, G. Ciavola, R. Harkewicz, K. Harrison, A. Srivastava, and P. Briand, Rev. Sci. Instrum. 67(12), 4109 (1996).

${ }^{5}$ C. M. Lyneis, D. Leitner, S. R. Abbott, R. D. Dwinell, M. Leitner, C. S. Silver, and C. Taylor, Rev. Sci. Instrum. 75, 1389 (2004).

${ }^{6}$ H. W. Zhao, L. T. Sun, X. H. Gou, X. Z. Zhang, Z. M. Zhang, P. Yuan, D. Z. Xie, J. Y. Li, Y. C. Feng, W. He, M. T. Song, X. X. Li, Y. Cao, H. Wang, B. H. Ma, W. Lu, W. L. Zhan, and B. W. Wei, High Energy Phys. Nucl. Phys. 31(Supp. I), 8 (2007).

${ }^{7}$ C. M. Lyneis, D. Leitner, D. S. Todd, G. Sabbi, S. Prestemon, S. Caspi, and P. Ferracin, Rev. Sci. Instrum. 79, 02A321 (2008).

${ }^{8}$ P. Ferracin, S. Caspi, H. Felice, D. Leitner, C. M. Lyneis, S. Prestemon, G. L. Sabbi, and D. S. Todd, Rev. Sci. Instrum. 81, 02 A309 (2010).

${ }^{9}$ P. Suominen, T. Ropponen, and H. Koivisto, Nucl. Instrum. Methods Phys. Res. A 578(2), 370 (2007).

${ }^{10}$ M. Inutake, T. Cho, M. Ichimura, K. Ishii, A. Itakura, I. Katanuma, Y. Kiwamoto, Y. Kusama, A. Mase, S. Miyoshi, Y. Nakashima, T. Saito, A. Sakasai, K. Sawada, I. Wakaida, N. Yamaguchi, and K. Yatsu, Phys. Rev. Lett. 55, 939 (1985).

${ }^{11}$ P. Suominen and F. Wenander, Rev. Sci. Instrum. 79, 02 A305 (2008).

${ }^{12}$ H. Koivisto, P. Heikkinen, V. Hänninen, A. Lassila, H. Leinonen, V. Nieminen, J. Pakarinen, K. Ranttila, J. Ärje, and E. Liukkonen, Nucl. Instrum. Methods Phys. Res. B 174, 379 (2001).

${ }^{13}$ P. Spädtke, Rev. Sci. Instrum. 75(5), 1643 (2004).

${ }^{14}$ P. Spädtke, Rev. Sci. Instrum. 81, $02 B 725$ (2010).

${ }^{15}$ P. Spädtke, KOBRA3-INP, INP, Junkernstr. 99, 65205 Wiesbaden, Germany, www.inp-dme.com.

${ }^{16} \mathrm{P}$. Spädtke, R. Lang, J. Mäder, J. Roßbach, and K. Tinschert, Proceedings of the 18th International Workshop on ECR Ion Sources (ECRISO8: JACOW), Chicago, IL, USA, 2009, THCO-BO2.

${ }^{17}$ Final Report of the EURISOL Design Study (2005-2009), edited by J. Cornell (GANIL, Caen, France, 2009). 